\title{
Comparison and Combination of Two Ultrasound Modalities, Handheld Ultrasound and Automated Breast Volume Scanner, With and Without Knowledge of MRI
}

\author{
Yoonsoo Kim, ${ }^{1}$ Bong Joo Kang, ${ }^{1,}{ }^{*}$ Sung Hun Kim, ${ }^{1}$ and Eun Jae Lee ${ }^{1}$ \\ ${ }^{1}$ Department of Radiology, Mary's Hospital, College of Medicine, The Catholic University of Korea, Seoul, Korea \\ "Corresponding author: Bong Joo Kang, Department of Radiology, Seoul St. Mary's Hospital, College of Medicine, The Catholic University of Korea, 222, Banpo-daero, Seocho-gu, \\ Seoul, Republic of Korea. Tel: +82-222586253, Fax: +82-25996771, E-mail: lionmain@catholic.ac.kr \\ Received 2017 November 02; Revised 2017 December 06; Accepted 2017 December 13.
}

\begin{abstract}
Background: When a suspicios breast lesion is found on MRI but is not observed on conventional imaging, targeted ultrasound of the MRI-detected lesion is commonly performed for its detection and characterization. Nonetheless, there are limitations of handheld ultrasound (HHUS). Another ultrasound modality named automated breast volume scanner (ABVS) has achieved automation and high resolution recently. The detection rate and accuracy of conjunctive and disjunctive combination of each ultrasound modality (HHUS and ABVS) have not yet been evaluated.

Objectives: To compare the diagnostic performance using HHUS, ABVS, and the combination method for suspicious lesions found on MRI.

Patients and Methods: From March to September on 2014, we prospectively enrolled 40 consecutive breast cancer patients who underwent HHUS and ABVS for newly detected suspicious lesions found on MRI. All patients underwent mammography and HHUS before MRI. Whole breast ABVS and another HHUS were performed after MRI. We reviewed the detection rate and diagnostic accuracy of each imaging and analyzed the conjunctive and disjunctive combination results of two ultrasound modalities. We then compared them with or without knowledge of MRI.

Results: In 120 suspicious lesions of 40 patients, seventy-six malignant and 44 nonmalignant lesions were included. With knowledge of MRI, cancer detection sensitivities of HHUS, ABVS, conjunctive and disjunctive combination were higher than those without knowledge of MRI (94.7\%, 98.7\%, 100\%, 93.4\% vs. 65.8\%, 64.5\%, 67.1\%, 63.2\%). In conjunctive combination of HHUS and ABVS, the detection sensitivities were improved in both cases with and without knowledge of MRI.

Conclusion: With knowledge of MRI, HHUS and ABVS imaging detected most lesions and the conjunctive combination showed the highest detection sensitivity.
\end{abstract}

Keywords: Breast Cancer, Magnetic Resonance Imaging, Ultrasound

\section{Background}

Although complete tumor resection is the optimum treatment for breast carcinoma, breast conservation surgery has recently become the standard operation and an increasingly common treatment for ductal carcinoma in situ (DCIS) and breast carcinoma (1-3). For determining the extent of the lesion and the presence of multifocal or multicentric lesions, patients with breast carcinoma undergo preoperative evaluations including breast magnetic resonance imaging (MRI), which has proven to be the most sensitive tool for the detection of breast carcinoma (4-7). Even though the use of preoperative breast MRI is increasing, controversy still exists for low specificity, unnecessary mastectomies, and cost-effectiveness (8). Recently, some studies have reported that MRI should be selectively used in patients with a maximum likelihood of additional information $(9,10)$.

When a lesion appears to be suspicious on an MRI image but is not observed on conventional imaging, targeted ultrasound imaging of the MRI-detected lesion is commonly performed for its detection and characterization. When the lesion has suspicious malignant features on targeted ultrasound, ultrasound-guided biopsy must be performed (11).

The relative limitations of handheld ultrasound (HHUS) are well documented, including high operatordependence and poor standardization of the technique, 
which can result in missed cancers and unnecessary biopsies, additional medical expenses, and patient anxiety. With the development of high-frequency transducers, the automated breast volume scanner (ABVS) has achieved automation and high resolution. Furthermore, automation of the ultrasound scanning has eliminated the problem of operator subjectivity and variation. The ABVS technique is reproducible and allows interpretation at a soft-copy display workstation, optimizing the radiologist's reading environment (12); however, the detection rate and accuracy of conjunctive and disjunctive combination of each ultrasound modality (HHUS and ABVS) have not yet been evaluated.

\section{Objectives}

The objective of our study was to compare the diagnostic performance using handheld ultrasound (HHUS), automated breast volume scanner (ABVS), and the combination method for the suspicious lesions found on MRI.

\section{Patients and Methods}

\subsection{Patient Population}

The Institutional review board approved this study and all enrolled patients provided informed consent. We prospectively enrolled breast cancer patients who underwent HHUS and ABVS scanning for newly detected lesions found via preoperative breast MRI. Between March 1, 2014, and September 30, 2014, 40 patients (120 total lesions) were enrolled in our study. The mean age of the 40 patients was 50.7 years (30 - 76 years). Of the study population, all patients underwent mammography and HHUS before the MR examinations. Whole breast ABVS and an additional HHUS scan were performed for the additionally detected suspicious lesions on MRI.

The final diagnosis was based on needle biopsy (ultrasound or MR-guided) or surgical result in most cases. Of these 120 lesions, 76 were malignant: 61 cases of invasive ductal carcinoma (IDC), eight cases of DCIS, four cases of mucinous carcinoma, two cases of invasive micropapillary carcinoma, and one case of invasive lobular carcinoma. In addition, 31 nonmalignant lesions were identified: eight fibrocystic changes, one fibroadenoma, 10 ductal hyperplasia, one stromal fibrosis, one fat necrosis, one columnar cell hyperplasia, two intraductal papilloma, two atypical ductal hyperplasia (ADH), four lobular neoplasia, and one flat epithelial atypia. No biopsy procedure (no 1:1 confirmation) was performed for the remaining 13 lesions. These lesions did not change or disappear by MRI follow-up (12 - 24 months) and were suggested to be benign (Table 1).

\begin{tabular}{|c|c|}
\hline Characteristics & Data \\
\hline Person age $(n=40)$ & Mean 50.7 (range $30-76)$ \\
\hline Lesion size $(n=120)$ & Mean $1.58 \mathrm{~cm}( \pm 1.64)$ \\
\hline & Pathology \\
\hline \multirow{7}{*}{ Benign (35) } & Fibrocystic changes (8) \\
\hline & Fibroadenoma (1) \\
\hline & Ductal hyperplasia (10) \\
\hline & Stromal fibrosis (1) \\
\hline & Fat necrosis (1) \\
\hline & Columnar cell hyperplasia (1) \\
\hline & No change by MR follow-up (13) \\
\hline \multirow{4}{*}{ Borderline, high-risk (9) } & Intraductal papilloma (2) \\
\hline & Atypical ductal hyperplasia (2) \\
\hline & Lobular neoplasia (4) \\
\hline & Flat epithelial atypia (1) \\
\hline \multirow{5}{*}{ Malignancy (76) } & Invasive ductal carcinoma (61) \\
\hline & Ductal carcinoma in situ (8) \\
\hline & Mucinous carcinoma (4) \\
\hline & Invasive micropapillary carcinoma (2) \\
\hline & Invasive lobular carcinoma (1) \\
\hline
\end{tabular}

\subsection{MRI Technique and Interpretation}

The MRI scans were acquired with the patient in the prone position in a 3.0 T Verio scanner (MagnetomVerio, Siemens Medical Solutions, Erlangen, Germany) with a breast coil. The following sequences were used in this study: axial, turbo spin-echo T2-weighted imaging (repetition time (TR)/echo time (TE) $4530 / 93 \mathrm{~ms}$, flip angle $80^{\circ}, 34$ slices with field of view (FOV) $320 \mathrm{~mm}$, matrix $576 \times 403,1$ number of excitations (NEX), $4 \mathrm{~mm}$ slice thickness, acquisition time 2 minutes 28 second). Pre- and post-contrast, axial, T1-weighted flash three dimensional (3D), volumetric interpolated breath-hold examination (VIBE) (TR/TE 4.4/1.7 $\mathrm{ms}$, flip angle $10^{\circ}, 1.2 \mathrm{~mm}$ slice thickness with no gap, acquisition time 60 second). The post-contrast scans were obtained at 7, 67, 127, 187, 247, and 367 seconds after injection of $0.1 \mathrm{mmol} / \mathrm{kg}$ gadopentetate dimeglumine (GdDPTA). MRI was interpreted based on the American college of radiology-breast imaging reporting and data system(ACR-BI-RADS)(13). Additionally detected suspicious lesion on MRI means above BI-RADS category 4. In this study, the breast cancer patients who had newly been detected with an additional suspicious lesion on MRI (above BI-RADS category 4 and BI-RADS category 6 [known cancer] lesions) were enrolled. 


\subsection{Mammography and Ultrasound Imaging Technique}

Conventional mammography with two views per breast was obtained using two units of Selenia (Hologic; Bedford, MA, USA) and Mammomat Inspiration (Siemens; Erlangen, Germany).

Handheld ultrasound (HHUS) was performed using two units of a 6- to 14-MHz linear-array transducer (EUB8500 scanner, Hitachi Medical, Tokyo, Japan) and a 7- to 15-MHz linear-array transducer (iU22 Ultrasound system, Philips Healthcare). An MRI-directed ("targeted") HHUS was performed an average of $3.6 \pm 2.0$ days (range, 1 - 10 days) after MRI by two expert breast radiologists with 10 and 12 years of experience. Radiological technologists were not involved in the HHUS studies.

Automated breast volume scanner (ABVS) was performed the same day as targeted HHUS. The scanning unit of ABVS contains a 5- to 14-MHz linear transducer (ACUSON S2000 ABVS system, Siemens Medical Solutions, Mountain View, CA, USA). This wide-aperture transducer can capture a volume of $17 \times 15 \times 6 \mathrm{~cm}^{3}$ in a single scan. The ABVS generates 318 2D slices with $0.5 \mathrm{~mm}$ thickness in the axialdirection single scan. The scan number required to image the whole breast without missing any parts depends on the patient's breast size. The diagnostic workstation unit of ABVS processes the 3D volume dataset in various multiplanar reconstructions and directions. In this study, axial, coronal-, and sagittal-direction scans were performed. A radiological technologist performed the ABVS scanning after receiving instruction concerning how to handle the equipment and completing a one-month training period. The scans of all 40 patients were interpreted by two expert breast radiologists with 10 and 12 years of experience. The radiologists reached a consensus without knowing the results of the targeted HHUS.

Mammogram and ultrasound without knowledge of MRI were interpreted based on the American College of Radiology-Breast imaging reporting and data system (ACRBI-RADS) $(13,14)$. In case of an additionally detected suspicious lesion on MRI, the detections on HHUS and ABUS were determined.

We reviewed each modality independently and evaluated the detection sensitivity, specificity, positive predictive value (PPV), and negative predictive value (NPV) of each modality. We analyzed the conjunctive and disjunctive combination results of two ultrasound modalities. In the conjunctive combination, non-detection on both the second HHUS and ABVS scans was defined as negative and detection on either the second HHUS or ABVS scans was defined as positive image. In the disjunctive combination, non-detection on either the second HHUS or ABVS scans was defined as negative and detection on the both the second HHUS and ABVS scans was defined as positive. We then compared them with or without knowledge of MRI. We correlated the results with the pathology results after the final surgical treatment. In addition, we compared the detected lesion size between MRI and the two ultrasound modalities.

\subsection{Data Analysis}

Patient information, including clinical data, imaging data and pathology results, was collected from the hospital's data systems. A statistical analysis of differences in the detection rate of the HHUS and ABVS techniques was performed using the McNemar test. Statistical analyses were conducted using the Chi-square test. The Fisher's exact test and Wilcoxon rank sum test were used to evaluate the association between MRI factors and targeted HHUS and ABVS scanning. We compared lesion size measured by MRI versus HHUS and ABVS scanning using Bland-Altman Graphs. All statistical analyses were performed using MedCalc version 12.7 (MedCalc Software, Mariakerke, Belgium) and the software package SAS Enterprise Guide 5.1 (SAS Institute, Inc, Cary, North Carolina).

\section{Results}

From March to September on 2014, both ABVS and HHUS scanning were performed for 40 patients with 120 suspicious lesions detected via breast MRI. Seventy-six malignant and 44 non-malignant lesions were included (Table 1).

Table 2 shows the cancer detection accuracy on conventional imaging methods with and without knowledge of MRI. With knowledge of MRI, the cancer detection sensitivities of HHUS, ABVS, and their conjunctive and disjunctive combinations were higher than those without knowledge of MRI $(94.7 \%, 98.7 \%, 100 \%$, and $93.4 \%$ vs. $65.8 \%, 64.5 \%$, $67.1 \%$, and $63.2 \%$ ) (Figure 1). In the conjunctive combination of HHUS and ABVS techniques, the sensitivities were improved in both cases with and without knowledge of MRI. In the disjunctive combination of HHUS and ABVS techniques, the specificities were improved in those with and without knowledge of MRI.

Without MRI, ultrasound detected lesions are larger than non-detected lesions (P value $<0.05$ ). Without MRI, DCIS showed a significant lower detection rate than invasive cancer (P value $<0.05$ ) (Table 3 ). With knowledge of MRI, HHUS and ABVS imaging detected most lesions, irrespective of size and DCIS (Table 3).

The size of the lesions on MRI correlated more with those detected using ABVS than HHUS (Figure 2). 

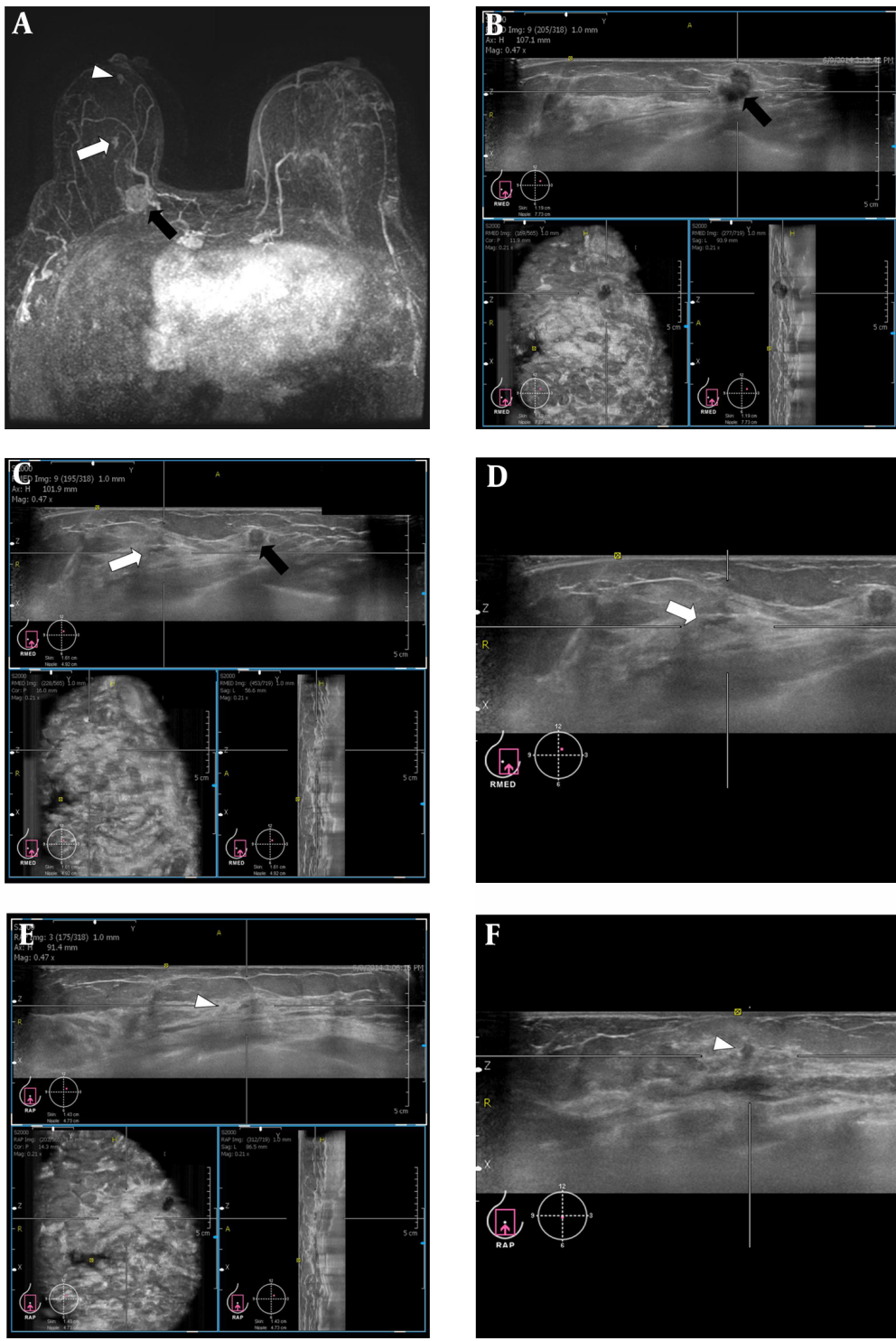

Figure 1. Images from a 68-year-old woman with right breast cancer (1-o'clock position, invasive ductal carcinoma (black arrow)) who underwent breast MRI for preoperative staging. A, Maximal intensity projection (MIP) reconstruction image showed two additional suspicious enhancing masses (white arrow and arrowhead) at the 1-o'clock position in the right breast. B, Three orthogonal planes (axial, sagittal, and coronal) of the right breast on the automated breast volume scanner (ABVS) revealed the known invasive ductal carcinoma (IDC) (black arrow). C, Three orthogonal planes (axial, sagittal, and coronal) of the right breast on the ABVS show the known IDC (black arrow) and an additional MRI detected suspicious lesion (white arrow). D, The axial plane of the right breast on the ABVS shows an additional MRI detected suspicious lesion confirmed as lobular neoplasia (white arrow). This lesion was not seen by handheld ultrasonography (US) imaging. E, Three orthogonal planes (axial, sagittal, and coronal) of the right breast on the ABVS show the other MRI detected suspicious lesion (white arrowhead). F, The axial plane of the right breast on the ABVS shows the additional MRI detected suspicious lesion confirmed as ductal carcinomas in situ (DCIS) (white arrowhead). This lesion was not seen by handheld US imaging. Surgical management was changed from breast-conserving surgery to a simple mastectomy.

\section{Discussion}

Breast MRI has been shown to be the most sensitive tool for the detection of breast cancer (4-7). Previous studies revealed that breast MRI is a reliable imaging modality for predicting tumor extension and for the detection of additional ipsilateral and contralateral tumor foci $(15,16)$. Exact treatment planning and accurate tumor removal is of the utmost importance (7).

Recently, breast-conserving surgery (BCS) for early breast cancer has proven to be as effective as conventional mastectomy in terms of the long-term survival rate and the recurrence rate (1-3). In addition, BCS is more esthetic than 
A

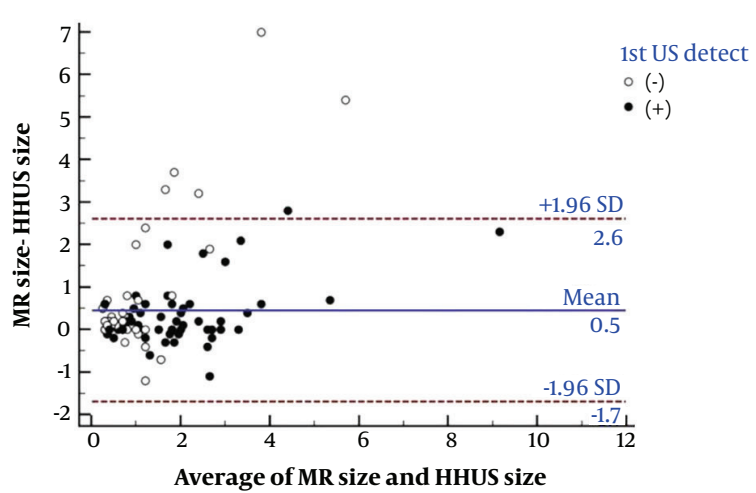

C

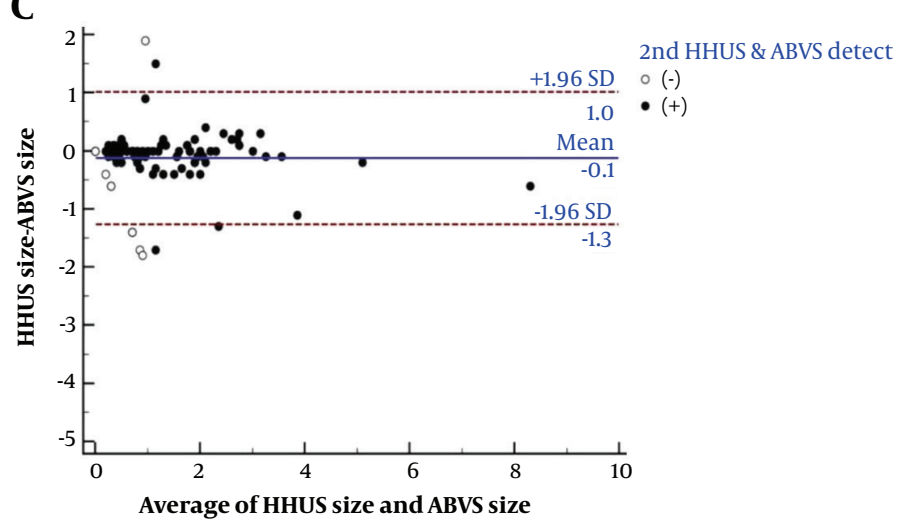

B

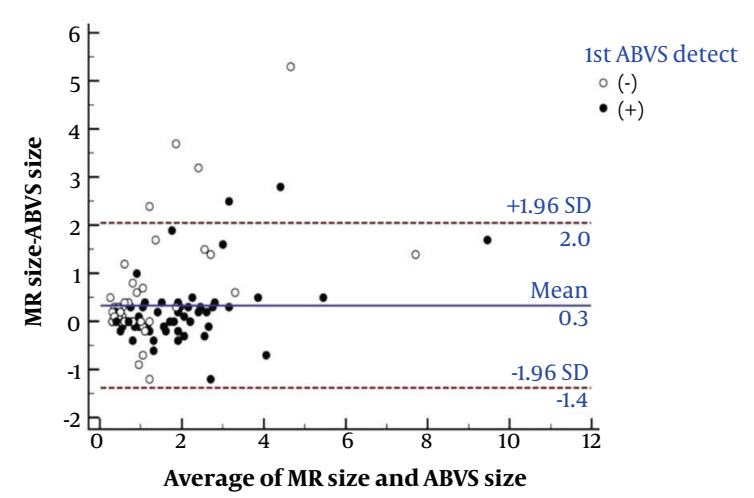




\begin{tabular}{|c|c|c|c|c|}
\hline Variables & Sensitivity & Specificity & PPV & NPV \\
\hline \multicolumn{5}{|l|}{ Pre-MRI (without MRI) } \\
\hline MMG (category ${ }^{\mathrm{a}}$ ) & 47.4 & 93.2 & 92.3 & 50.6 \\
\hline Fatty breast $(1+2)$ & 60.0 & 90.5 & 85.7 & 70.4 \\
\hline Dense breast $(3+4)$ & 42.9 & 95.7 & 96.0 & 40.7 \\
\hline 1st HHUS detection ${ }^{\mathrm{b}}$ & 65.8 & 59.1 & 73.5 & 50.0 \\
\hline ABVS detection ${ }^{\mathrm{b}}$ & 64.5 & 68.2 & 77.8 & 52.6 \\
\hline Conjunctive ${ }^{c}$ combination detection & 67.1 & 56.8 & 72.9 & 50.0 \\
\hline Disjunctive $^{\mathrm{d}}$ combination detection & 63.2 & 75.0 & 81.4 & 54.1 \\
\hline 1st HHUS category ${ }^{\mathrm{a}}(\mathrm{n}=68)$ & 56.6 & 100.0 & 100.0 & 57.1 \\
\hline ABVS category $^{\mathrm{a}}(\mathrm{n}=63)$ & 59.2 & 97.7 & 97.8 & 58.1 \\
\hline \multicolumn{5}{|l|}{ Post-MRI (with MRI) } \\
\hline $1 \mathrm{st}+2$ nd HHUS detection ${ }^{\mathrm{b}}$ & 94.7 & 13.6 & 65.5 & 60.0 \\
\hline ABVS detection ${ }^{\mathrm{b}}$ & 98.7 & 6.8 & 64.7 & 75.0 \\
\hline Conjunctive ${ }^{c}$ combination & 100.0 & 6.8 & 65.0 & 100.0 \\
\hline Disjunctive $^{\mathrm{d}}$ combination & 93.4 & 13.6 & 65.1 & 54.6 \\
\hline \multicolumn{5}{|c|}{$\begin{array}{l}\text { Abbreviations: ABVS, automated breast volume scanner; HHUS, handheld ultrasound; MMG, mammography; NPV, negative predictive value; PPV, positive predictive } \\
\text { value. } \\
\text { a Category: category 1, 2, and } 3 \text { were defined as negative category (-), 4, 5, and o were defined as positive category (+) given to MMG, 1st HHUS category, ABVS category, and } \\
\text { MRI. } \\
\text { b Detection: non-detection was defined as negative (-), detection was defined as positive (+) given to HHUS, ABVS, conjunctive and disjunctive combination detections. } \\
\text { cConjunctive combination: non-detection; non-detection on both the } 2 \text { nd HHUS and ABVS scans; detection; detection on either the } 2 \text { nd HHUS or ABVS scans. } \\
\text { d Disjunctive combination: non-detection; non-detection on either the } 2 \text { nd HHUS or ABVS scans; detection; detection on the both the } 2 \text { nd HHUS and ABVS scans. }\end{array}$} \\
\hline
\end{tabular}

already suspicious on MRI. Based on the known fact that the specificity for breast MRI is variable, our result showing low specificity on ultrasonography (USG) has a different meaning from the specificity of ultrasound alone. In this study, the breast cancer patients who had newly been detected with an additional suspicious lesion on MRI (above BI-RADS category 4 and BI-RADS category 6 [known cancer] were enrolled. The patients who had proven cancer(s) with/without non-suspicious lesions on MRI (BI-RADS category 6) were not included. MRI had high sensitivity and found a lot of suspicious looking lesions later proven benign and borderline lesions. With knowledge of MRI, the rates of non-detected lesion on ABVS and HHUS were very low. So, the specificities of HHUS and ABVS with knowledge of MRI could be low. The detection sensitivity of ultrasound on the MRI-detected lesions had the most significant value. In addition, because of the suspiciously malignant findings on the MRI image, MRI-detected lesions were not evaluated by BI-RADS ultrasound categories.

Nevertheless, if a suspicious finding on an MRI image is found, a subsequent biopsy should be performed for preoperative staging; however, MRI-guided biopsy is expensive, time-consuming, and uncomfortable for the patient (11). Targeted ultrasound may visualize and further characterize these lesions and facilitate ultrasound-guided biopsy when correlated with MRI findings (21). Therefore, when abnormalities detected by MRI lack visualization on conventional imaging (mammograms or first breast ultrasound), a targeted or MRI-directed ultrasound is commonly performed to further characterize the MRI findings (22).

The role of targeted ultrasound has been investigated by several authors (11, 21, 23-29). Previous studies showed that the frequency of which MRI-detected lesions are found by ultrasound ranges from $23 \%$ to $89 \%$. The mean detection rate of ultrasound was $63 \%$. Carbognin et al. documented that more than $90 \%$ of lesions $>10 \mathrm{~mm}$ were detected on ultrasound when compared with lesions that were $10 \mathrm{~mm}$ or less. Wiratkapun et al. reported a statistically significant direct association between increasing MRI mass lesion size and the ultrasound detection rate (odds ratio $=$ 1.23). In this study, ultrasound without knowledge of MRI showed a lower detection rate for small-sized lesions ( $\mathrm{P}$ value $<0.05$ ). However, ultrasound with knowledge of MRI showed no significant association between the detection rate and lesion size.

In this study, the detection rate using ABVS was higher than that using HHUS (98.7\% vs. 94.7\%, P < 0.05) with 


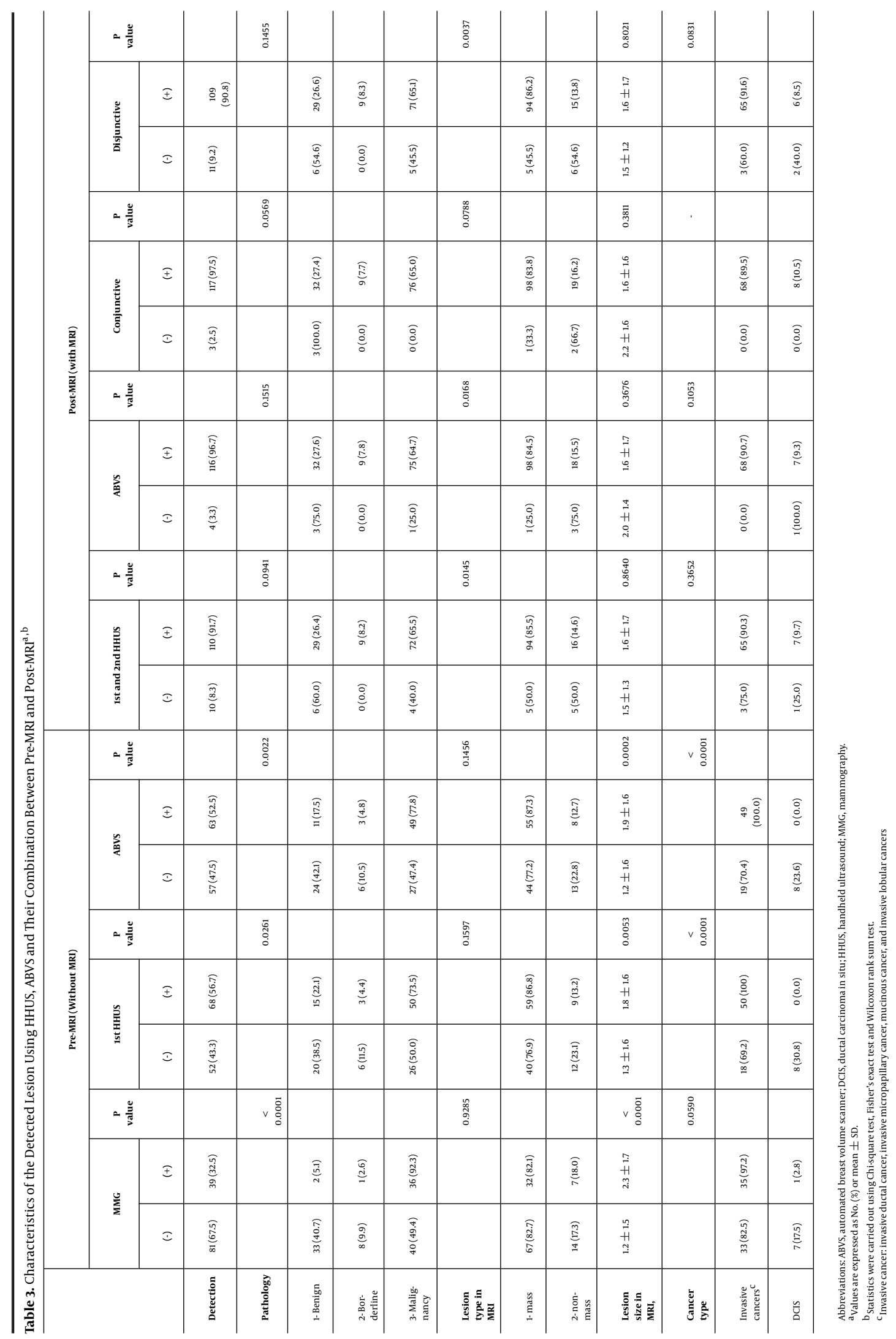


knowledge of MRI, demonstrating the advantage of ABVS over HHUS scanning for standardized, reproducible, and bilateral whole-breast imaging. In contrast to HHUS, which is a real-time examination, the reader can review the whole images scanned by ABVS reproducibly. This advantage could reduce the number of missed diagnoses. The ABVS also provides a more accurate view than HHUS for evaluating breast masses because each cross-sectional plane of the scanned images can be visualized. The ability to scan a 3D volume of both breasts allows the images to be reviewed irrespectively of the location and time of the actual examination. These characteristics make the ABVS a promising diagnostic tool for targeted ultrasound. Additionally, the size of the lesions on MRI correlated more with those detected using ABVS than HHUS.

However, the utility of real-time imaging by HHUS to determine the mobility of the lesion is an important feature that is not available with the ABVS. Moreover, detection on HHUS is needed for an ultrasound-guided biopsy. If the initial ultrasound was performed by ABVS and a targeted ultrasound is necessary, a re-review of the ABVS images could be useful without requiring the patient to return for a targeted HHUS examination. In conjunctive combination of HHUS and ABVS techniques, the detection sensitivities were improved in both cases with and without knowledge of MRI. In disjunctive combination of HHUS and ABVS techniques, the specificities were improved with and without knowledge of MRI.

Our study was limited by the small study population size. Furthermore, we only enrolled lesions detected on MRI so we could not judge other lesions that might be missed in MRI. However, to our knowledge, this report was the first study to analyze the clinical utility, which includes detection rate and diagnostic accuracy of the combined use of ABVS and HHUS for MRI-detected lesions.

With knowledge of MRI, HHUS and ABVS imaging detected most lesions and the conjunctive combination of HHUS and ABVS showed the highest sensitivity. The sensitivity of ABVS was higher than HHUS, and the size of the lesions on MRI correlated more with those detected using ABVS than HHUS. Therefore, after preoperative MRI, ABVS imaging could be suggested for optimally targeted ultrasound methods. Then, the HHUS technique could be selected for ultrasound-guided biopsy or additional targeted methods.

In conclusion, the role of MRI for preoperative assessment is irreplaceable. With knowledge of MRI, the conjunctive combination of HHUS and ABVS techniques showed the highest sensitivity. In additional, ABVS imaging is better for preoperative evaluation than HHUS.

\section{Acknowledgments}

The authors wish to acknowledge financial support from the catholic Medical center research foundation during the 2014 program year. The statistical consultation was supported by a grant of the Korean health technology $\mathrm{R}$ and D project, ministry of health and welfare, Republic of Korea (HI14C1731).

\section{Footnotes}

Authors' Contributions: Study concept and design, Bong Joo Kang; analysis and interpretation of data, Yoonsoo Kim, and Bong Joo Kang; drafting of the manuscript, Yoonsoo Kim; critical revision of the manuscript for important intellectual content, Yoonsoo Kim, Bong Joo Kang, Sung Hun Kim, and Eun Jae Lee; statistical analysis, Yoonsoo Kim, and Bong Joo Kang.

Financial Disclosure: The authors declare that there are no conflicts of interest.

Funding/Support: The authors wish to acknowledge financial support from the catholic Medical center research foundation during the 2014 program year. The statistical consultation was supported by a grant of the Korean health technology R and D project, ministry of health and welfare, Republic of Korea (HI14C1731).

\section{References}

1. Fisher B, Anderson S, Bryant J, Margolese RG, Deutsch M, Fisher ER, et al. Twenty-year follow-up of a randomized trial comparing total mastectomy, lumpectomy, and lumpectomy plus irradiation for the treatment of invasive breast cancer. NEngl J Med. 2002;347(16):1233-41. doi 10.1056/NEJMoa022152. [PubMed: 12393820].

2. Poggi MM, Danforth DN, Sciuto LC, Smith SL, Steinberg SM, Liewehr DJ, et al. Eighteen-year results in the treatment of early breast carcinoma with mastectomy versus breast conservation therapy: the National Cancer Institute Randomized Trial. Cancer. 2003;98(4):697-702. doi: 10.1002/cncr.11580. [PubMed: 12910512].

3. Veronesi U, Cascinelli N, Mariani L, Greco M, Saccozzi R, Luini A, et al. Twenty-year follow-up of a randomized study comparing breastconserving surgery with radical mastectomy for early breast cancer. N Engl J Med. 2002;347(16):1227-32. doi: 10.1056/NEJMoa020989. [PubMed: 12393819].

4. Berg WA, Gutierrez L, NessAiver MS, Carter WB, Bhargavan M, Lewis $\mathrm{RS}$, et al. Diagnostic accuracy of mammography, clinical examination, US, and MR imaging in preoperative assessment of breast cancer. $R a-$ diology. 2004;233(3):830-49. doi: 10.1148/radiol.2333031484. [PubMed: 15486214].

5. Kuhl C. The current status of breast MR imaging. Part I. Choice of technique, image interpretation, diagnostic accuracy, and transfer to clinical practice. Radiology. 2007;244(2):356-78. doi: 10.1148/radiol.2442051620. [PubMed: 17641361].

6. Kuhl CK. The "coming of age" of nonmammographic screening for breast cancer. JAMA. 2008;299(18):2203-5. doi: 10.1001/jama.299.18.2203. [PubMed: 18477789]. 
7. Debald M, Abramian A, Nemes L, Dobler M, Kaiser C, Keyver-Paik $\mathrm{MD}$, et al. Who may benefit from preoperative breast MRI? A singlecenter analysis of 1102 consecutive patients with primary breast cancer. Breast Cancer Res Treat. 2015;153(3):531-7. doi: 10.1007/s10549-0153556-3. [PubMed: 26323190].

8. Gonzalez V, Sandelin K, Karlsson A, Aberg W, Lofgren L, Iliescu G, et al. Preoperative MRI of the breast (POMB) influences primary treatment in breast cancer: a prospective, randomized, multicenter study. World J Surg. 2014;38(7):1685-93. doi: 10.1007/s00268-014-2605-0. [PubMed: 24817517].

9. Fortune-Greeley AK, Wheeler SB, Meyer AM, Reeder-Hayes KE, Biddle AK, Muss HB, et al. Preoperative breast MRI and surgical outcomes in elderly women with invasive ductal and lobular carcinoma: a population-based study. Breast Cancer Res Treat. 2014;143(1):203-12. doi: 10.1007/s10549-013-2787-4. [PubMed: 24305978].

10. Pengel KE, Loo CE, Wesseling J, Pijnappel RM, Rutgers EJ, Gilhuijs KG. Avoiding preoperative breast MRI when conventional imaging is sufficient to stage patients eligible for breast conserving therapy. Eur J Radiol. 2014;83(2):273-8. doi: 10.1016/j.ejrad.2013.10.018. [PubMed: 24220546].

11. Abe H, Schmidt RA, Shah RN, Shimauchi A, Kulkarni K, Sennett CA, et al. MR-directed ("Second-Look") ultrasound examination for breast lesions detected initially on MRI: MR and sonographic findings. AJR Am J Roentgenol. 2010;194(2):370-7. doi: 10.2214/AJR.09.2707. [PubMed: 20093598].

12. Kuzmiak CM, Ko EY, Tuttle LA, Steed D, Zeng D, Yoon SC. Whole Breast Ultrasound: Comparison of the Visibility of Suspicious Lesions with Automated Breast Volumetric Scanning Versus Hand-Held Breast Ultrasound. Acad Radiol. 2015;22(7):870-9. doi: 10.1016/j.acra.2015.03.006. [PubMed: 25872862].

13. Radiology. ACo . Breast imaging reporting and data system, breast imaging atlas. 5th ed. American College of Radiology; 2013.

14. Eberl MM, Fox CH, Edge SB, Carter CA, Mahoney MC. BI-RADS classification for management of abnormal mammograms. J Am Board Fam Med. 2006;19(2):161-4. doi: 10.3122/jabfm.19.2.161. [PubMed: 16513904].

15. Plana MN, Carreira C, Muriel A, Chiva M, Abraira V, Emparanza JI, et al. Magnetic resonance imaging in the preoperative assessment of patients with primary breast cancer: systematic review of diagnostic accuracy and meta-analysis. Eur Radiol. 2012;22(1):26-38. doi: 10.1007/s00330-011-2238-8. [PubMed: 21847541].

16. Liberman L, Morris EA, Dershaw DD, Abramson AF, Tan LK. MR imaging of the ipsilateral breast in women with percutaneously proven breast cancer. AJR Am J Roentgenol. 2003;180(4):901-10. doi: 10.2214/ajr.180.4.1800901. [PubMed: 12646427].

17. Bellon JR, Harris EE, Arthur DW, Bailey L, Carey L, Goyal S, et al. ACR Appropriateness Criteria( $R$ ) conservative surgery and radiation-stage I and II breast carcinoma: expert panel on radiation oncology: breast. Breast J. 2011;17(5):448-55. doi: 10.1111/j.1524-4741.2011.01132.x. [PubMed: 21790842].
18. Theriault RL, Carlson RW, Allred C, Anderson BO, Burstein HJ, Edge $\mathrm{SB}$, et al. Breast cancer, version 3.2013: featured updates to the NCCN guidelines. J Natl Compr Canc Netw. 2013;11(7):753-60. quiz 761. doi: 10.6004/jnccn.2013.0098. [PubMed: 23847214].

19. Sardanelli F, Boetes C, Borisch B, Decker T, Federico M, Gilbert FJ, et al. Magnetic resonance imaging of the breast: recommendations from the EUSOMA working group. Eur J Cancer. 2010;46(8):1296-316. doi: 10.1016/j.ejca.2010.02.015. [PubMed: 20304629].

20. Hollingsworth AB, Stough RG. Preoperative breast MRI for locoregional staging. J Okla State Med Assoc. 2006;99(10):505-15. [PubMed: 17125104].

21. LaTrenta LR, Menell JH, Morris EA, Abramson AF, Dershaw DD, Liberman L. Breast lesions detected with MR imaging: utility and histopathologic importance of identification with US. Radiology. 2003;227(3):856-61. doi: 10.1148/radiol.2273012210. [PubMed: 12773685].

22. Ferre R, Ianculescu V, Ciolovan L, Mathieu MC, Uzan C, Canale $S$, et al. Diagnostic Performance of MR-guided Vacuum-Assisted Breast Biopsy: 8 Years of Experience. Breast J. 2016;22(1):83-9. doi: 10.1111/tbj.12519. [PubMed: 26511082].

23. Sim LS, Hendriks JH, Bult P, Fook-Chong SM. US correlation for MRI-detected breast lesions in women with familial risk of breast cancer. Clin Radiol. 2005;60(7):801-6. doi: 10.1016/j.crad.2004.12.005. [PubMed: 15978891].

24. Beran L, Liang W, Nims T, Paquelet J, Sickle-Santanello B. Correlation of targeted ultrasound with magnetic resonance imaging abnormalities of the breast. Am J Surg. 2005;190(4):592-4. doi: 10.1016/j.amjsurg.2005.06.019. [PubMed: 16164928].

25. Wiratkapun C, Duke D, Nordmann AS, Lertsithichai P, Narra V, Barton PT, et al. Indeterminate or suspicious breast lesions detected initially with MR imaging: value of MRI-directed breast ultrasound. Acad Radiol. 2008;15(5):618-25. doi: 10.1016/j.acra.2007.10.016. [PubMed: 18423319].

26. Demartini WB, Eby PR, Peacock S, Lehman CD. Utility of targeted sonography for breast lesions that were suspicious on MRI. AJR Am J Roentgenol. 2009;192(4):1128-34. doi: 10.2214/AJR.07.3987. [PubMed: 19304724].

27. Destounis S, Arieno A, Somerville PA, Seifert PJ, Murphy P, Morgan $\mathrm{R}$, et al. Community-based practice experience of unsuspected breast magnetic resonance imaging abnormalities evaluated with second-look sonography. J Ultrasound Med. 2009;28(10):1337-46. doi: 10.7863/jum.2009.28.10.1337. [PubMed: 19778880].

28. Meissnitzer M, Dershaw DD, Lee CH, Morris EA. Targeted ultrasound of the breast in women with abnormal MRI findings for whom biopsy has been recommended. AJR Am J Roentgenol. 2009;193(4):1025-9. doi: 10.2214/AJR.09.2480. [PubMed: 19770325].

29. Shin JH, Han BK, Choe YH, Ko K, Choi N. Targeted ultrasound for MR-detected lesions in breast cancer patients. Korean J Radiol. 2007;8(6):475-83. doi:10.3348/kjr.2007.8.6.475. [PubMed: 18071277]. 